\title{
Optimal Feedback for Quality Source-Adaptive Schemes in Multicast Multi-layered Video Environments ${ }^{\star}$
}

\author{
Paulo André da Silva Gonçalves ${ }^{1,2}$, José Ferreira de Rezende ${ }^{2}$, \\ Otto Carlos Muniz Bandeira Duarte ${ }^{2}$, and Guy Pujolle ${ }^{1}$ \\ 1 LIP6 - Université Pierre et Marie Curie \\ 8, rue du Capitaine Scott - 75015 - Paris \\ \{Paulo.Goncalves, Guy.Pujolle\}@lip6.fr \\ 2 GTA/COPPE - Universidade Federal do Rio de Janeiro \\ P.O. Box 68504 - 21945-970 - Rio de Janeiro - RJ - Brazil \\ $\{$ rezende, otto\}@gta.ufrj.br
}

\begin{abstract}
Current quality source-adaptive schemes for multicast multilayered video rely on merging capabilities at special nodes in the network as a means of combining feedback from the whole set of receivers. These strategies reduce network load and avoid feedback implosion at the source. In this paper, we examine how to provide optimal feedback for such schemes. The optimal feedback is achieved when state information from the whole set of receivers is represented in every incoming feedback packet at the source. We show that the choice of a suitable merging time window in the intermediate nodes coupled with a periodical transmission of feedback packets by the receivers leads to near-optimal feedback.
\end{abstract}

\section{Introduction}

The need for meeting the Quality of Service (QoS) requirements of the multimedia applications is steadily increasing. As a consequence, new approaches such as adaptive applications 1, QoS routing [2, 3] and Differentiated Services [4], [5] are currently being developed. In the case of adaptive applications, different schemes are emerging to improve quality and fairness of multi-layered video applications [6], 7], 8], [9], [10, 11], 12], [13], [14]. In the context of unicast, as an example, Rejaie et al. 8], 9] propose an adaptation scheme in which the perceived video quality at the receiver is more stable even in the presence of fluctuations in the available network bandwidth. In this scheme, the receiver plays an important role by buffering video layers and sending to the source feedback packets containing current network congestion information.

In the context of multicast, exchanging network congestion information or more generally any kind of information between receivers and source is a nontrivial matter. The challenge facing this approach is twofold. First, the source

\footnotetext{
* This work was sponsored by: CAPES/Brazil, COFECUB, CNRS, and CNPq. Paulo André da Silva Gonçalves has a scolarship from CAPES/Brazil.
} 
will be prone to feedback implosion. Second, even if feedback implosion avoidance mechanisms are employed, a means of allowing that the incoming feedback at the source represents the current state of the whole set of receivers should be provided. In the contrary, i.e. in the case of the source has only a partial state information, the source adaptation may be unsuitable and unfair when regarding receivers whose state is not included in feedback packets. If every incoming feedback at the source represents the state information from the whole set of receivers in a multicast group, optimal feedback is achieved.

In this paper, we investigate how to provide optimal feedback for current quality source-adaptive schemes in multicast multi-layered video environments. The efficiency of such schemes in terms of quality/fairness, network load and source implosion depends on the choice of three different policies: feedback transmission, temporal-merging and content-merging policies. The first policy is related to when receivers should send feedback packets to the source. The second one determines when feedback packets are merged by intermediate nodes. The last policy establishes how feedback packets are merged. The effectiveness of the feedback transmission and temporal-merging policies, which are investigated in this paper, dictates the efficiency of the third policy.

The paper is organized as follows. In Section 2, we explain the motivation for this work by presenting current quality source-adaptive schemes proposed in the literature. In Section 3, we describe the temporal-merging policy and analyze in which conditions this policy provides optimal feedback. The formulas that model the temporal-merging policy are presented in Section 4 . We thereafter analyze how optimal feedback is related to feedback suppression. In Section 5] we provide a feedback transmission policy that coupled with the temporal-merging policy leads to an optimal feedback. Theoretical and simulation results are presented in Section 6. Finally, in Section 7 we conclude the paper.

\section{Motivation}

The Receiver-driven Layered Multicast (RLM) 6] scheme pioneered the study of the multicast multi-layered video transmission. In RLM, each video layer is sent out on a different multicast group. Based on congestion control information, receivers can subscribe to a number of layers that can be supported by their individual path from the source. The Layered Video Multicast with Retransmissions (LVMR) [7] employs a hierarchical rate control to manage the adding and dropping of video layers by receivers. Furthermore, LVMR deploys an error recovery mechanism using retransmissions to adapt to network congestion. Despite the quality adaptation being provided by the schemes cited above, receivers are limited to the layers the source decides to transmit. Current quality source-adaptive schemes provide different solutions to overcome this limitation.

In [11], Vickers et al. propose a source-adaptive multi-layered multicast algorithm in which feedback control packets containing current network congestion information are exchanged between source and receivers. In this algorithm, the source periodically sends to the multicast group a control packet that is updated 
with congestion control information as it traverses the branches of the multicast tree. Upon receiving a control packet, the receiver returns to the source a feedback packet containing the received congestion control information. Based on the goodput quality metric, intermediate nodes combine feedback packets' contents in order to estimate the number of video layers and their respective transmission rates. Another scheme for adapting the quality of multi-layered video is proposed in 15. The control information at intermediate nodes is similar to the approach in [11. However, different from other proposals, content-merging procedures are performed in a single loop and the concept of virtual layers is employed. The result is an improvement in the fairness of the delivered service.

The classical approach to compute the rates of the layers for source-adaptive multi-layered multicast schemes is based on the goodput quality metric. This approach is analyzed in [13. The authors have demonstrated that the classical approach fails in the computation of the most adequate rates for the layers. In another work [10], the same authors propose another scheme for quality adaptation. Such a scheme is based on a combined metric that allows for the density of satisfied users, the weighted bandwidth allocation, and the goodput quality at the receivers. The proposed scheme yields enhancements in quality/fairness of multi-layered multicast sessions.

The quality source-adaptive schemes we mentioned earlier rely on the same temporal-merging policy performed at intermediate nodes. However, such schemes focus on how to efficiently combine packets' contents to yield improvements in the delivered service. We argue that the efficiency of such schemes can be maximized if optimal feedback is provided.

\section{Temporal-Merging Policy}

Consider our basic network topology as shows Fig. 1 The feedback-merger capable-node waits for packets from $k$ receivers. The temporal-merging policy is performed as follows. Incoming feedback packets are merged and forwarded upstream if one of the following two conditions holds. (i) At least one feedback packet from each node has arrived. (ii) The merger timer expires. If more than one feedback packet from the same node arrives before merging, the previous stored packet is replaced.

The basic strategy to achieve an optimal feedback is to maximize the number of packet merges. In this way, as regarding the temporal-merging policy described above, we should provide a means of minimizing the number of packet replacements and maximizing the number of packet merges due to condition (i). Providing such a means can ultimately be regarded as finding a feedback transmission policy and a merging time window that will allow to achieve an optimal feedback. 


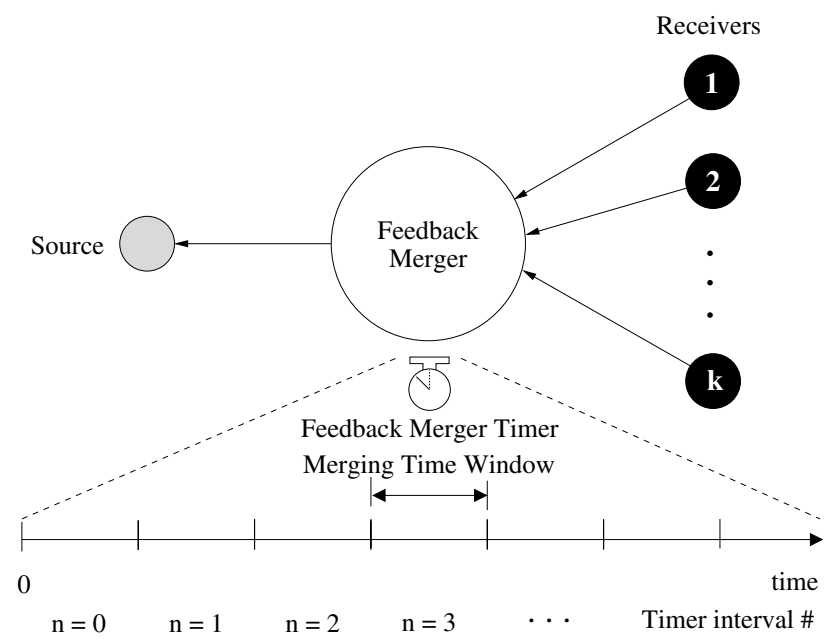

Fig. 1. Basic network topology and merger timer schematic

\section{Temporal-Merging Policy Model}

In this section, we first describe a formula to compute the number of merges due to conditions (i) and (ii), and next we analyze how optimal feedback is related to feedback suppression. In our analysis, we consider that the merger timer expires in a periodical fashion. Another possible approach would be to reschedule the timer upon arriving the first candidate packet for merging. Despite this approach only implies changes in the conditions for which formulas presented throughout this section hold, the effects of such approach are not evaluated in this paper.

\subsection{Merges Due to Conditions (i) and (ii)}

Consider the merger timer schematic shown in Fig. 1 For a given timer interval $n$, let $\rho_{i}^{n}$ and $\beta_{i}^{n}$ be, respectively, the number of packets arrivals from node $i$ and the number of packet replacements for nodes $i$. Then, for a feedback merger waiting for packets from $k$ nodes, the number of outcoming and incoming packets in the timer interval $n$ is, respectively, given by

$$
\gamma_{\text {out }}^{n}=\max _{i=1 \ldots k}\left[\rho_{i}^{n}-\beta_{i}^{n}\right]
$$

and

$$
\gamma_{i n}^{n}=\sum_{i=1}^{k} \rho_{i}^{n} .
$$

Given a timer interval $n$, the number of outcoming packets due to condition (i) and condition (ii) is, respectively, given by

$$
\mu_{k}^{n}=\min _{i=1 \ldots k}\left[\rho_{i}^{n}-\beta_{i}^{n}\right]
$$


and

$$
\sigma_{k}^{n}=\max _{i=1 \ldots k}\left[\rho_{i}^{n}-\beta_{i}^{n}\right]-\min _{i=1 \ldots k}\left[\rho_{i}^{n}-\beta_{i}^{n}\right] .
$$

If upon expiring the merging timer there exists at least one feedback packet waiting for merging then $\sigma_{k}^{n}=1$ else $\sigma_{k}^{n}=0$. Note that the number of outcoming packets in any timer interval $n$ is equal to $\mu_{k}^{n}+\sigma_{k}^{n}$ that provides the same result as in (1).

Considering $N$ timer intervals, the ratio of outcoming packets due to each condition to the number of incoming packets is, respectively, given by

$$
\theta_{k}^{\operatorname{cond}(i)}=\frac{\sum_{n=0}^{N-1} \mu_{k}^{n}}{\sum_{n=0}^{N-1} \gamma_{i n}^{n}} \quad 0 \leq \theta_{k}^{\operatorname{cond}(i)} \leq 1
$$

and

$$
\theta_{k}^{\operatorname{cond}(i i)}=\frac{\sum_{n=0}^{N-1} \sigma_{k}^{n}}{\sum_{n=0}^{N-1} \gamma_{i n}^{n}} \quad 0 \leq \theta_{k}^{\operatorname{cond}(i i)} \leq 1 .
$$

These formulas hold if for some $0 \leq n \leq(N-1)$ and $1<i \leq k$, there exists a $\gamma_{i n}^{n}$ such that $\gamma_{i n}^{n} \geq 1$. The fraction of merges due to condition (i) and condition (ii) is, respectively, given by

$$
\alpha_{k}^{\operatorname{cond}(i)}=\frac{\sum_{n=0}^{N-1} \min _{i=1 \ldots k}\left[\rho_{i}^{n}-\beta_{i}^{n}\right]}{\sum_{n=0}^{N-1} \max _{i=1 \ldots k}\left[\rho_{i}^{n}-\beta_{i}^{n}\right]} \quad 0 \leq \alpha_{k}^{\operatorname{cond}(i)} \leq 1
$$

and

$$
\alpha_{k}^{\operatorname{cond}(i i)}=1-\frac{\sum_{n=0}^{N-1} \min _{i=1 \ldots k}\left[\rho_{i}^{n}-\beta_{i}^{n}\right]}{\sum_{n=0}^{N-1} \max _{i=1 \ldots k}\left[\rho_{i}^{n}-\beta_{i}^{n}\right]} \quad 0 \leq \alpha_{k}^{\operatorname{cond}(i i)} \leq 1 .
$$

These formulas hold if for some $0 \leq n \leq(N-1)$ and $1<i \leq k$, there exists a $\rho_{i}^{n}$ such that $\rho_{i}^{n} \geq 1$. In Section 6 we will use these formulas to find a merging time window that minimizes the fraction of merges due to condition (ii). Since $\alpha_{k}^{\operatorname{cond}(i i)}$ is the complementary value of $\alpha_{k}^{\operatorname{cond}(i)}$, the minimization of the fraction of merges due to condition (ii) implies the maximization of the fraction of merges due to condition (i). From this fact, minimization of $\alpha_{k}^{c o n d(i i)}$ and maximization of $\alpha_{k}^{\text {cond(i) }}$ are interchangeable throughout this paper.

\subsection{Feedback Suppression}

The notion of a high level of feedback suppression is commonly employed as a measure of efficiency for implosion avoidance. Let us then verify how optimal feedback is related to feedback suppression. We define the Filtering Level $\left(\xi^{n}\right)$ in the timer interval $n$ as the number of packets suppressed by the feedback merger over the total number of incoming packets, that is,

$$
\xi^{n}=1-\frac{\gamma_{o u t}^{n}}{\gamma_{\text {in }}^{n}} \quad 0 \leq \xi^{n}<1
$$




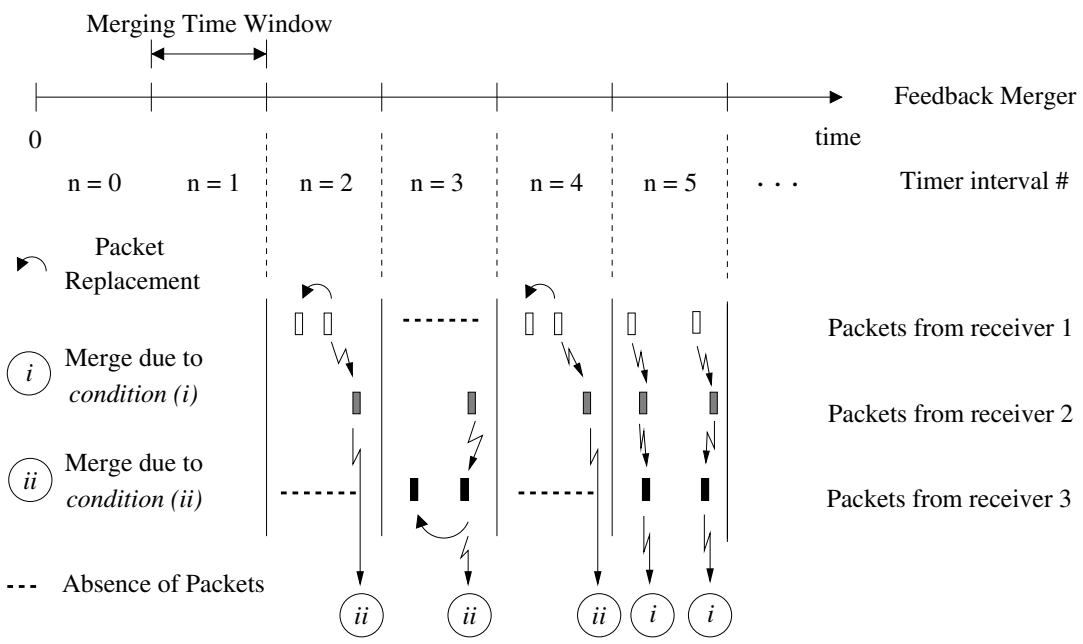

Fig. 2. Scenario in which the Global Filtering Level assumes its optimal value

if $\gamma_{i n}^{n} \geq 1$. In generalizing our definition to take into account a number $N$ of timer intervals, we get the Global Filtering Level

$$
\xi_{k}=1-\frac{\sum_{n=0}^{N-1} \gamma_{o u t}^{n}}{\sum_{n=0}^{N-1} \gamma_{i n}^{n}} \quad 0 \leq \xi_{k}<1 .
$$

Substituting equations (11) and (2) in (10), we get

$$
\xi_{k}=1-\frac{\sum_{n=0}^{N-1} \max _{i=1 \ldots k}\left[\rho_{i}^{n}-\beta_{i}^{n}\right]}{\sum_{n=0}^{N-1} \sum_{i=1}^{k} \rho_{i}^{n}} \quad \text { for } k>1
$$

and if for some $0 \leq n \leq(N-1)$ and $1 \leq i \leq k$, there exists a $\rho_{i}^{n}$ such that $\rho_{i}^{n} \geq 1$. If $k \leq 1$ then $\xi_{k}=0$.

Now let us find the optimal value for $\xi_{k}$. For this purpose, consider both the network example as depicted in Fig. 1] and the following scenario where optimal feedback occurs. For every timer interval $n$ having packets within there are no packet replacements and the number of packet arrivals from all nodes is the same. Thus, for any timer interval $n$ having packets within, the ratio $\gamma_{\text {out }}^{n} / \gamma_{\text {in }}^{n}$ will always be constant and equal to $1 / k$. Without loss of generality, the ratio $\sum_{n=0}^{N-1} \gamma_{\text {out }}^{n} / \sum_{n=0}^{N-1} \gamma_{i n}^{n}$ will also be equal to $1 / k$. In this manner, the optimal value for $\xi_{k}$ is given by

$$
\xi_{k, \text { optimal }}=1-\frac{1}{k} \quad \text { for } k>1 .
$$

A necessary condition to achieve an optimal feedback is that the Global Filtering Level be as close as possible of its optimal value. However, an optimal value of the Global Filtering Level does not imply optimal feedback because packet replacements may be occurring as well as merges due to condition (ii). 
This situation is illustrated in Fig. 2. Considering the timer intervals from 2 to 5, the Global Filtering Level $\left(\xi_{3}\right)$ is equals to its optimal value of 0.66 .

Other important remarks are the following. If there are no packet replacements, $\xi_{k}$ will be less or equal to $\xi_{k, \text { optimal }}$. If, on the other hand, there are packet replacements, $\xi_{k}$ may assume values greater than $\xi_{k, \text { optimal }}$. If $\alpha_{k}^{\text {cond(ii) is }}$ equal to 0 then $\xi_{k}$ will be equal or greater than $\xi_{k \text {,optimal }}$. In this case, $\xi_{k}$ will be equal to $\xi_{k, \text { optimal }}$ if there are no packet replacements and it will be greater than $\xi_{k, o p t i m a l}$ if there is at least one packet replacement. Thus, despite a Global Filtering Level $\left(\xi_{k}\right)$ greater than its optimal value impacts better the efficiency for feedback implosion avoidance, it is not adequate to an effective feedback.

\section{Choice of the Feedback Transmission Policy}

Both the number of packets and the number of packet replacements in each timer interval depend on the feedback transmission policy. When a number of feedback packets must be sent from receivers to the source, packet replacements can be minimized if we allow the arrival rate of feedback from the different receivers at a feedback merger be the same. In practice, it is a nontrivial matter to ensure that the arrival rate of feedback be the same because feedback packets may suffer from random delays into the network and be lost. However, a first approximation is to assign to all receivers the same feedback sending interval.

Since we defined a feedback transmission policy, let us now provide means of quantifying $\rho_{i}^{n}$. For this purpose, recall our basic network topology and consider both schematics the feedback transmission and the merger timer expiration as depicted in Fig. 3. The merger timer expires in a periodical fashion at time instants $\tau_{e x p}^{(n)}$ given by (13), where $n=0,1,2, \ldots$ is the current timer interval, $\Delta \tau$ is the merging time window size, and $\delta^{(n)}$ is a random variable representing jitter in the timer interval $n$.

$$
\tau_{\text {exp }}^{(n)}= \begin{cases}\Delta \tau+\delta^{(n)} & \text { if } n=0 \\ \tau_{\text {exp }}^{(n-1)}+\Delta \tau+\delta^{(n)} & \text { otherwise }\end{cases}
$$

As depicted in Fig. [3, each receiver $i$ transmits feedback packets in a periodical fashion. The arrival time $\tau_{\text {arrival, } i}^{\left(r_{i}\right)}$ of a feedback packet at the feedback merger is given by (14), where $r_{i}=0,1,2, \ldots$ is the current sending round number for receiver $i, \Delta t$ represents the feedback sending interval and $\sigma_{i}^{\left(r_{i}\right)}$ models processing time delay in the sending round $r_{i}$. The start-time of a receiver $i$ is denoted by $c_{i}$. Queuing delay in a given sending round $r_{i}$, transmission delay and propagation delay are, respectively, denoted by $Q_{i}^{\left(r_{i}\right)}, T_{i}$ and $P_{i}$.

$$
\tau_{\text {arrival }, i}^{\left(r_{i}\right)}= \begin{cases}c_{i}+Q_{i}^{\left(r_{i}\right)}+P_{i}+T_{i} & \text { if } r_{i}=0 \\ \tau_{\text {arrival }, i}^{\left(r_{i}-1\right)}+\Delta t_{i}+\sigma_{i}^{\left(r_{i}\right)}+Q_{i}^{\left(r_{i}\right)}+P_{i}+T_{i} & \text { otherwise }\end{cases}
$$

In (14), we assume that there is no packet loss and the transmission buffer at nodes is infinite. Feedback packets that are candidates to be merged must arrive 


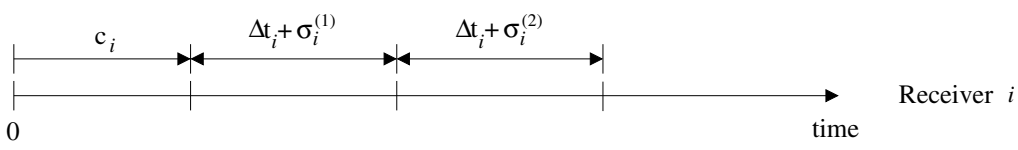

$$
\begin{array}{lllll}
\mathrm{r}_{i}=0 & \mathrm{r}_{i}=1 & \mathrm{r}_{i}=2 & \ldots & \text { Sending Round \# }
\end{array}
$$

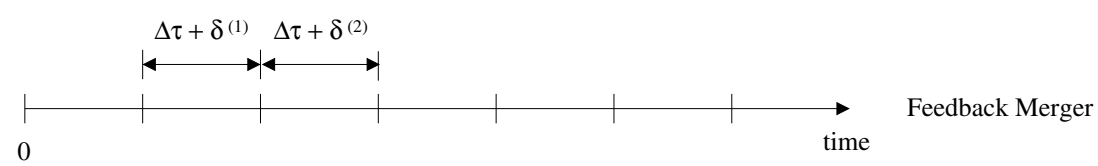

$\mathrm{n}=0 \quad \mathrm{n}=1 \quad \mathrm{n}=2 \quad \mathrm{n}=3 \quad \cdots \quad$ Timer interval \#

Fig. 3. Feedback transmission policy and merger timer expiration schematics

at the feedback merger between two consecutive timer expirations. Thus, based on (13) and (14) we can write the following.

$$
h \tau_{\text {exp }}^{(n-h)} \leq \tau_{\text {arrival }, i}^{\left(r_{i}-m\right)}+m\left(\Delta t_{i}+\sigma_{i}^{\left(r_{i}\right)}+Q_{i}^{\left(r_{i}\right)}+P_{i}+T_{i}\right)<\tau_{\text {exp }}^{(n h)},
$$

where $m$ is equal to 0 if $r_{i}=0$ and equal to 1 otherwise and $h$ is equal to 0 if $n=0$ and equal to 1 otherwise. For a given timer interval $n$, let $\Upsilon_{i}^{n}$ be the set of elements $r_{i} \in \mathbb{N}$ for which the inequalities above are respected. Now let $\mathfrak{N}\left(\Upsilon_{i}^{n}\right)$ be the number of elements in $\Upsilon_{i}^{n}$. Then, the number of packets from node $i$ in any timer interval $n$ is $\rho_{i}^{r}=\mathfrak{N}\left(\Upsilon_{i}^{n}\right)$.

\section{Results}

In this section we present our theoretical and simulation results.

\subsection{Theoretical}

We assume that feedback packets from different receivers arrive at the feedback merger on different incoming links. A contrary assumption only impacts the minimal merging time window value that can be chosen. Finding such a value is outside of the scope of this paper. We set $\delta^{(n)}, \sigma_{i}^{\left(r_{i}\right)}$, and $Q_{i}^{\left(r_{i}\right)}$ to zero in order to assess the isolated behavior of the temporal-merging policy coupled with our choosen feedback transmission policy. In this manner, we will be able to find a merge time window that ensures $100 \%$ of merges due to condition (i) at steady state.

Respectively for $k=2$ and $k=3$, Figs. 4(a) and 4(b) show the behavior at steady state of the pair $\left(\xi_{k}, \alpha_{k}^{\operatorname{cond}(i i)}\right)$ as both the feedback sending interval and the deviation among receivers' start-time vary. Note that for a given $\Delta t$, different pairs $\left(\xi_{k}, \alpha_{k}^{\operatorname{cond}(i i)}\right)$ come from the different deviations among receivers' starttime. Packet replacements were not observed. The main results are the following. 
First, merges due to condition (ii) lead to a Global Filtering Level less than its optimal value, and second, assigning the merging time window to a multiple of the feedback sending interval ensures $100 \%$ of merges due to condition (i), regardless of receivers' start-time. That is, packet merges are maximized for $\Delta \tau=b \Delta t$, where $b=1,2, \ldots$, and as a consequence, optimal feedback is provided to the source. Since the maximum waiting time for merging feedback packets is directly proportional to the factor $b$, we adopt its minimum value, i.e. $b=1$.

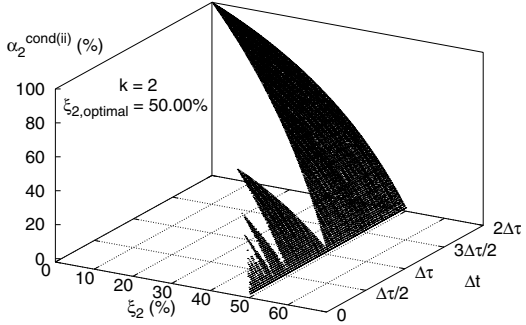

(a)

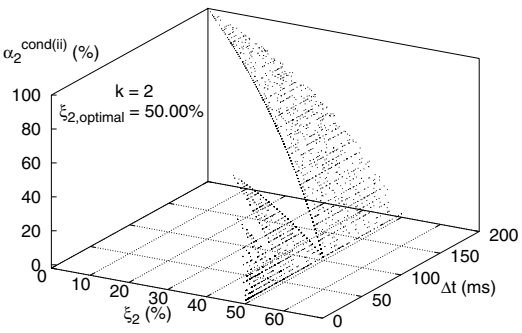

(c)

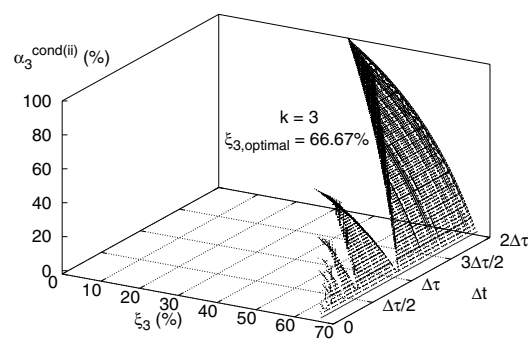

(b)

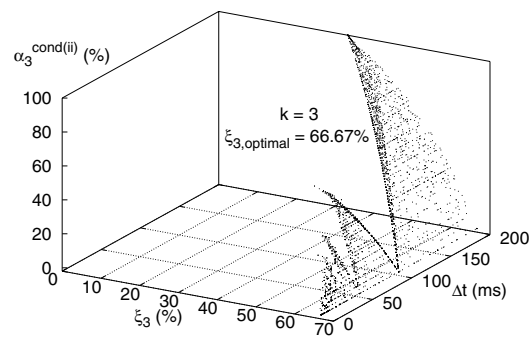

(d)

Fig. 4. Theoretical and simulation results

\subsection{Simulation}

We validate our theoretical results through simulation using ns-2 [16], [17]. Figs. 4(c) and 4(d) show the results we obtained under the same conditions earlier described in this section. Let us now verify how the previous results are impacted in a heterogeneous network with unpredictable random delays. Fig. 5 
shows the transit-stub network topology used in our simulations. Link capacities are 10 Mbps. Each link delay was randomly chosen uniformly on the interval $[1,10] \mathrm{ms}$. All intermediate nodes are capable of merging feedback packets. The merging time window and the feedback sending interval were set to $500 \mathrm{~ms}$. Background traffic is generated on the transit domain throughout all simulation time. For each sending round, processing delay at receivers was uniformly chosen at random on the interval $[0,5] \mathrm{ms}$. Merging time window variation in each merging procedure was randomly chosen uniformly on the interval $[0,5] \mathrm{ms}$. The start-time of each receiver was randomly chosen uniformly on the interval $[0,1]$ s. Each simulation was run for 315 seconds. All simulation results have a confidence interval of $90 \%$.

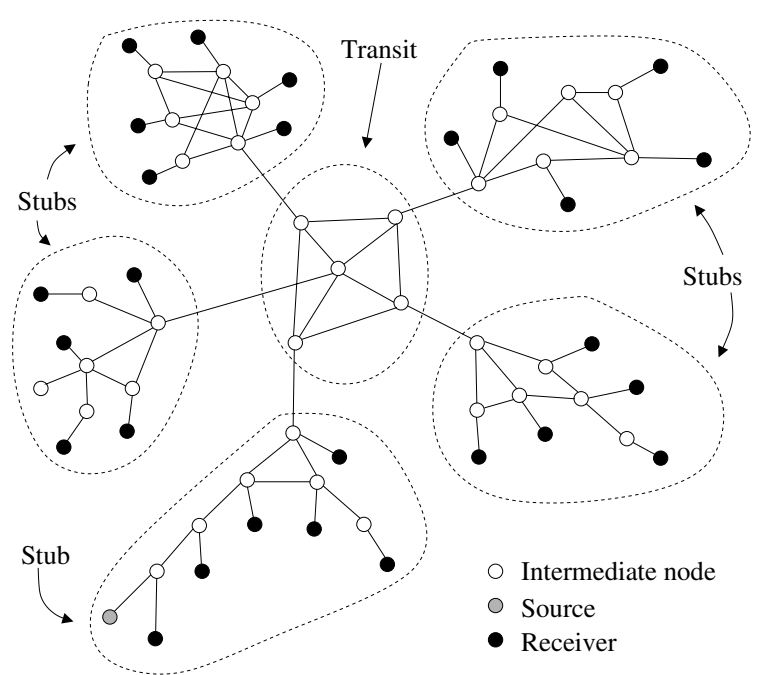

Fig. 5. Simulation Topology

The Global Filtering Level and the fraction of merges due to condition (i) were analyzed varying the feedback sending interval. Packet replacements were less than $0.4 \%$ for the feedback sending interval equals to the merging time window size. As regarding the others feedback sending intervals up to $5 \%$ of packet replacements were observed.

Fig.6(a) shows a Global Filtering Level varying from $94.30 \%$ to $96.16 \%$, making evident the effectiveness of the temporal-merging policy for feedback implosion avoidance. The Global Filtering Level achieves a maximum value of $96.16 \%$ when the merging time window size is assigned to the value of the feedback sending interval. In this case, the Global Filtering Level tended to its optimal value. For the others feedback sending intervals, the Global Filtering Level did not exceed this maximum value. That comes from the following fact. If on the one hand merges due to condition (ii) lead to a Global Filtering Level less than 
its optimal value, on the other hand, packet replacements lead to a Global Filtering Level greater than its optimal value. Thus, merges due to condition (ii) had a greater impact on the Global Filtering Level than packet replacements.

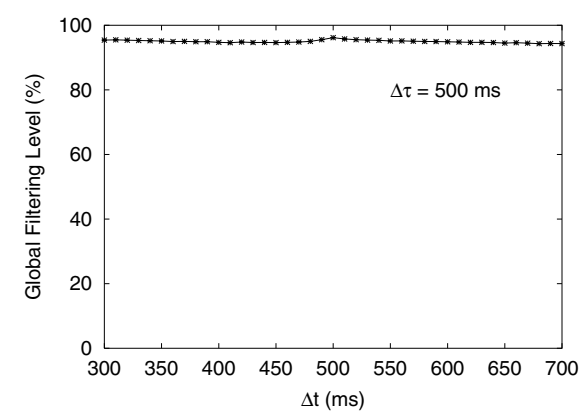

(a)

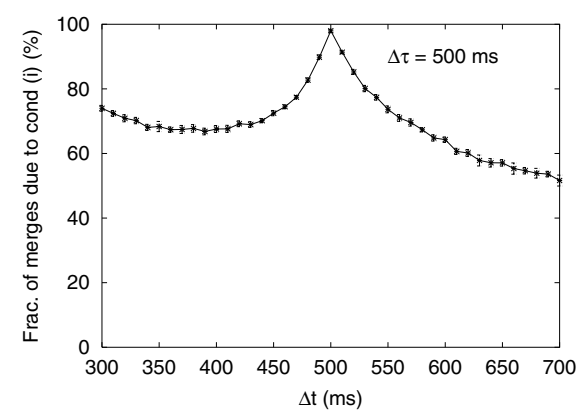

(b)

Fig. 6. Global Filtering Level and fraction of merges due to condition (i)

Fig. 6(b) shows how the merges occur into the network as the feedback sending interval is varied. In particular, around $2 \%$ of merges occur due to condition (ii) when the merging time window is assigned to the value of the feedback sending interval. This result shows that unpredictable random delays negatively impacts the fraction of merges due to condition (i), and as a consequence, only near-optimal feedback can be provided to the source. Nevertheless, this negative effect can be minimized if feedback mergers employ an adaptive merging time window.

\section{Conclusion}

In this paper, we have addressed the issue of providing optimal feedback for quality source-adaptive schemes in multicast multi-layered video environments. We argued that while current quality source-adaptive schemes focus on how to combine efficiently packets' contents to yield improvements in the delivered service, they are incapable of achieving their maximal efficiency if optimal feedback is not provided. We investigated how optimal feedback is related to feedback suppression. Our analysis showed that there exists an optimal value for the packet filtering level. Higher values lead to non-optimal feedback despite they imply efficiency for feedback implosion avoidance. We also demonstrated that by sending feedback packets in a periodical fashion and assigning the merging time window to the sending interval of feedback, near-optimal feedback can be achieved. 


\section{References}

1. C. Diot, C. Huitema, and T. Turletti, "Multimedia applications should be adaptive," in HPCS'95, (Mystic, CN), Aug. 1995.

2. Z. Wang and J. Crowcroft, "Quality of service routing for supporting multimedia applications," IEEE Journal on Selected Areas in Communications, vol. 14, Sept. 1996.

3. L. H. M. K. Costa, S. Fdida, and O. C. M. B. Duarte, "A scalable algorithm for link-state QoS-based routing with three metrics," in IEEE ICC'2001, (Helsink, Filand), June 2001.

4. S. Blake et al., An Architecture for Differentiated Services. Internet RFC 2475, Dec. 1998.

5. A. Ziviani, J. F. de Rezende, O. C. M. B. Duarte, and S. Fdida, "Evaluating voice traffic in a differentiated services environment," in Proceedings of the 17th International Teletraffic Congress - ITC17, (Salvador, Brazil), Dec. 2001.

6. S. McCanne, V. Jacobson, and M. Vetterli, "Receiver-driven layered multicast," in SIGCOMM'96, (Stanford, CA), pp. 117-130, Aug. 1996.

7. X. Li, S. Paul, and M. H. Ammar, "Layered video multicast with retransmission (LVMR): Evaluation of hierarchical rate control," in IEEE INFOCOM'98, (San Francisco, CA), pp. 1062-1072, Apr. 1998.

8. R. Rejaie, D. Estrin, and M. Handley, "Quality adaptation for congestion controlled video playback over the internet," in Proceedings of ACM SIGCOMM'99, (Cambridge, MA), Sept. 1999.

9. R. Rejaie, M. Handley, and D. Estrin, "Layered quality adaptation for internet video streaming," IEEE Journal on Selected Areas in Communications, vol. 18, Dec. 2000.

10. M. D. de Amorim, O. C. M. B. Duarte, and G. Pujolle, "Application-Aware multicast," in IEEE GLOBECOM'2001, (San Antonio, Texas), Nov. 2001.

11. B. J. Vickers, C. Albuquerque, and T. Suda, "Adaptive multicast of multi-layered video: Rate-based and credit-based approachs," in IEEE INFOCOM'98, (San Francisco, CA), Apr. 1998.

12. P. A. da Silva Gonçalves, J. F. de Rezende, and O. C. M. B. Duarte, "An active service for multicast video distribution," Journal of the Brazilian Computer Society, vol. 7, pp. 43-51, July 2000.

13. M. D. de Amorim, O. C. M. B. Duarte, and G. Pujolle, "Multi-criteria arguments for improving the fairness of layered multicast applications," in Lecture Notes in Computer Science, no. 1815, pp. 1-10, May 2000.

14. B. J. Vickers, M. Lee, and T. Suda, "Feedback control mechanisms for real-Time multipoint video services," IEEE Journal on Selected Areas in Communications, vol. 15, pp. 512-530, Apr. 1997.

15. M. D. Amorim, O. C. M. B. Duarte, and G. Pujolle, "Single-loop packet merging for receiver oriented multicast multi-layered video," in International Conference in Computer Communications, (Tokyo, Japan), Sept. 1999.

16. K. Fall and K. Varadhan, "NS notes and documentation," tech. rep., The VINT Project, July 1999.

17. S. Bajaj et al., "Improving simulation for network research," tech. rep., University of Southern California, Department of Computer Science, Mar. 1999. 